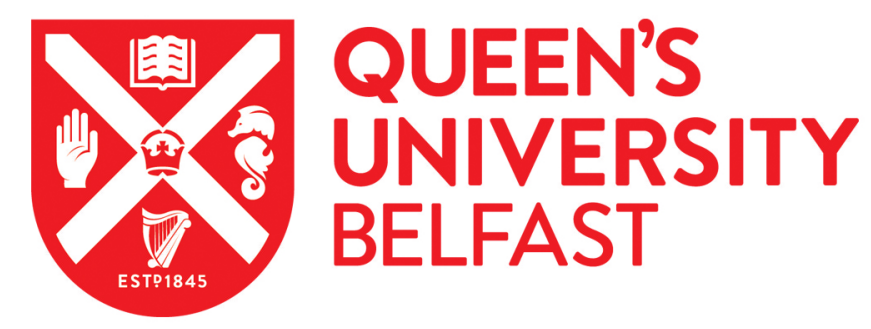

\title{
Intracellular Activation of a Prostate Specific Antigen-Cleavable Doxorubicin Prodrug: A Key Feature Toward Prodrug-Nanomedicine Design
}

Pereira, S. G. T., Hudoklin, S., Kreft, M. E., Kostevsek, N., Stuart, M. C. A., \& Al-Jamal, W. T. (2019).
Intracellular Activation of a Prostate Specific Antigen-Cleavable Doxorubicin Prodrug: A Key Feature Toward
Prodrug-Nanomedicine Design. Molecular Pharmaceutics. https://doi.org/10.1021/acs.molpharmaceut.8b01257

Published in:

Molecular Pharmaceutics

Document Version:

Peer reviewed version

Queen's University Belfast - Research Portal:

Link to publication record in Queen's University Belfast Research Portal

Publisher rights

( 2019 American Chemical Society. This work is made available online in accordance with the publisher's policies. Please refer to any applicable terms of use of the publisher

\section{General rights}

Copyright for the publications made accessible via the Queen's University Belfast Research Portal is retained by the author(s) and / or other copyright owners and it is a condition of accessing these publications that users recognise and abide by the legal requirements associated with these rights.

Take down policy

The Research Portal is Queen's institutional repository that provides access to Queen's research output. Every effort has been made to ensure that content in the Research Portal does not infringe any person's rights, or applicable UK laws. If you discover content in the Research Portal that you believe breaches copyright or violates any law, please contact openaccess@qub.ac.uk. 
Intracellular Activation of a Prostate Specific Antigen-Cleavable Doxorubicin Prodrug: a Key Feature Towards Prodrug-Nanomedicine Design

Sara G. T. Pereira ${ }^{\mathrm{a}}$, Samo Hudoklin ${ }^{\mathrm{b}}$, Mateja Erdani Kreft ${ }^{\mathrm{b}}$, Nina Kostevsek ${ }^{\mathrm{c}}$, Marc C. A. Stuart $^{\mathrm{d}}$, Wafa T. Al-Jamal ${ }^{*}$

${ }^{a}$ School of Pharmacy, Queen's University Belfast, 97 Lisburn Rd, Belfast BT9 7BL

${ }^{\mathrm{b}}$ University of Ljubljana, Faculty of Medicine, Institute of Cell Biology, Ljubljana, Slovenia

${ }^{\mathrm{c}}$ Department for Nanostructured Materials, Jozef Stefan Institute, Ljubljana, Slovenia

${ }^{\mathrm{d}}$ Electron Microscopy, University of Groningen, Nijenborgh 7, 9747AG Groningen, The Netherlands

*Corresponding author:

Dr Wafa' T. Al-Jamal

School of Pharmacy

Queen's University Belfast

Belfast, BT9 7BL

United Kingdom

E-mail: w.al-jamal@qub.ac.uk 


\begin{abstract}
L-377,202 prodrug (Dox-PSA) was in phase I Clinical trial for patients with metastatic castration-resistant prostate cancer (mCRPC). It consists of doxorubicin (Dox) conjugated to a prostate specific antigen (PSA)-cleavable peptide that can be selectively activated by secreted PSA at the tumour site. However, despite the initial promising results, further clinical testing with Dox-PSA was halted due to toxicity concerns emerging from non-PSA-specific cleavage, following systemic administration. In the present study, we have reported, and for the first time, the intracellular activation of DoxPSA, where Dox nuclear uptake was specific to C4-2B (PSA-expressing) cells, which agreed with the cytotoxicity studies. This finding was confirmed by encapsulating DoxPSA prodrug into $\mathrm{pH}$-sensitive liposomes to enable prodrug intracellular release, followed by its enzymatic activation. Interestingly, our results demonstrated that DoxPSA loaded into $\mathrm{pH}-$ responsive nanoparticles exhibited cytotoxicity comparable to free prodrug in C4-2B monolayers, with superior activity in tumour spheroids, due to deeper penetration within tumour spheroids. Our approach could open the doors for novel DoxPSA nanomedicines with higher safety and efficacy to treat advanced and metastatic prostate cancer.
\end{abstract}

Keywords: prostate cancer, prostate specific antigen-cleavable doxorubicin prodrug, $\mathrm{pH}-$ sensitive liposomes, intracellular activation 


\section{Introduction}

Despite the recent advances in the treatment of metastatic castration-resistant prostate cancer (mCRPC), its clinical outcome is still poor as an effective therapy remains an unmet need ${ }^{1}$. $\mathrm{mCRPC}$ is initially managed with castration therapy but inevitably, 18 to 36 months after therapy initiation, hormone- and chemoresistant cells emerge ${ }^{2}$. Bypassing chemoresistance and dose-limiting systemic toxicity has urged the development of alternative therapeutic modalities such as prodrugs. These pharmaceuticals are characterised by the covalent modification of the parental drug with a promoiety that renders the compound pharmacologically inactive until certain conditions are encountered ${ }^{3,4}$. Once in the in vivo milieu, the prodrug undergoes enzymatic and/or chemical bioconversion steps that transform it into the biologically active form ${ }^{5}$. Dox is an anthracycline antibiotic which acts by intercalating with DNA therefore inhibiting topoisomerase II and causing double-strand DNA breaks and cell death. Despite Dox wide spectrum of anticancer activity in both solid and liquid tumours, the emergence of drug resistance and cardiotoxicity limit its clinical use, factors that could be ameliorated through a prodrug approach ${ }^{6,7}$. In the past decade, doxorubicin (Dox) prodrugs have been designed to be specifically metabolised by prostate specific antigen (PSA) in prostate cancer (PC). PSA is a serine protease with a physiological function of liquefying the sperm coagulum by specifically cleaving its natural substrates semenogelins I and $\mathrm{II}^{8}$. The relevance of PSA as a therapeutic target is based on the premise that the PSA found in the tumour microenvironment is catalytically active, whilst the PSA that leaks into the blood is inactivated by its covalent binding to serum protease inhibitors, primarily $\alpha 1-$ antichymotrypsin and $\alpha 2$-macroglobulin ${ }^{9}$. Several PSA-specific peptide sequences have been developed, including Ser-Lys-Leu-Gln, His-Ser-Ser-Lys-Leu-Gln and Ser-Ser-LysTyr-Gln ${ }^{10}$. A common feature among all these sequences is the presence of a Gln residue at position $\mathrm{P}_{1}$, imparting PSA-specificity as PSA is the only member of the kallikrein family involved in the proteolytic cleavage of substrates having a Gln residue in that position ${ }^{11}$. Conjugation of the PSA substrates to a wide variety of cytotoxic drugs ${ }^{10}$ has been accomplished by using the dipeptide Ser-Leu or Leu as a linker to improve PSAhydrolysis rate. Other PSA substrates were developed comprising non-natural amino acids in their peptide sequence in order to increase the chemical stability of the peptidedrug conjugate and mitigate hydrolysis by non-PSA proteases. One such example is the peptide-drug conjugate Glutaryl-Hyp-Ala-Ser-Chg-Gln-Ser-Leu-Dox (L-377,202- 
hereafter called Dox-PSA) which, despite having reach the clinical setting for mCRPC, did not advance beyond phase I trials due to toxicity concerns ${ }^{12,13}$. Although in preclinical studies in mice, rats, dogs and monkeys, Dox-PSA selectively accumulated in the tumour and the concentrations of Dox in the cardiac tissue (site of clinical dose-limiting toxicity) were much lower than those achieved when Dox was administered alone, there was 30$40 \%$ of non-PSA-specific metabolism of Dox-PSA ${ }^{14}$. Moreover, while in the preclinical setting administration of the prodrug into nude mice bearing LNCaP xenografts greatly improved the therapeutic index, with 8- to 9-fold molar increase in the dose of Dox administered $^{15}$, the clinical scenario was largely dissimilar, where only 1.5 -fold more molar equivalent of Dox could be administered. Additionally, 1 of 6 patients treated with Dox-PSA at the efficacious dose of $225 \mathrm{mg} / \mathrm{m}^{2}$ exhibited dose-limiting neutropenia ${ }^{16}$. These results would likely limit the therapeutic efficacy of this prodrug and therefore new strategies urge development before Dox-PSA can be used as an effective therapy for mCRPC.

Aiming at improving the specificity of Glutaryl-Hyp-Ala-Ser-Chg-Gln as a PSAcleavable promoiety, Aloysius et al. have recently designed the sequences Glutaryl-SerAla-Ser-Chg-Gln and GABA-mGly-Ala-Ser-Chg-Gln. The authors have shown that while substitutions between $\mathrm{P}_{1}$ and $\mathrm{P}_{4}$ would adversely impact on PSA specificity, the given modifications at $\mathrm{P}_{5}$ improved PSA-mediated hydrolysis rate and decreased nonPSA-specific cleavage ${ }^{13}$. In the present work, we sought to explore a versatile approach where, rather than altering Dox-PSA prodrug design, we relied on its encapsulation inside a delivery system capable of improving Dox-PSA enzymatic stability. By revisiting DoxPSA and transforming it into a macromolecular prodrug, we aim not only to alter the pharmacokinetics and pharmacodynamics of the prodrug, but also protect it from the premature proteolytic cleavage while in the bloodstream ${ }^{17,18}$ which, overall, could contribute to an increased therapeutic efficacy. An additional benefit of this nanoparticlebased approach is the improved tumour targeting that might be achieved by exploring the enhanced permeability and retention (EPR) effect, therefore evading a potential nonPSA-specific prodrug activation in healthy tissues. Importantly, unlike small molecules that can freely diffuse across cellular membranes, nanotherapeutics can only enter the cell by endocytosis ${ }^{19}$. A direct consequence of this is that our proposed approach would necessarily rely on Dox-PSA intracellular activation. However, to the best of our knowledge, previous studies with PSA-cleavable prodrugs have invariably grounded on the extracellular activation of the prodrug due to the secretory nature of PSA enzyme and 
the notion that high intracellular zinc concentrations inhibit PSA activity ${ }^{12,13,20}$. Yet, the dysregulation of several zinc transporters has been associated with low intracellular zinc levels in malignant prostate epithelial cells ${ }^{21,22}$. Therefore, the main goal of the present work is to investigate if Dox-PSA can be intracellularly activated and maintain its cytotoxic activity. If these premises hold truth, such a provocative and sophisticated strategy could speed up the clinical translation of Dox-PSA. In a proof-of-concept study, and to eliminate the possibility of extracellular activation, Dox-PSA was encapsulated into $\mathrm{pH}$-sensitive liposomes composed of phosphatidylethanolamine (DOPE) and cholesteryl hemisuccinate (CHEMS), which could destabilise the endosomal membrane and release their cargos to the cytosol, thereby bypassing lysosomal degradation ${ }^{23-25}$. Interestingly, our results revealed for the first time that the intracellular delivery of DoxPSA via DOPE:CHEMS liposomes resulted in high cytotoxicity in two- and threedimensional PC models, confirming the intracellular activation of Dox-PSA. Our strategy holds great promise to improve the therapeutic efficacy of Dox-PSA and to avoid non-specific Dox-PSA activation and consequent dose-limiting toxicity in vivo following systemic administration.

\section{Experimental Section}

\section{Chemicals}

PSA-cleavable peptide (Glutaryl-4-Hyp-Ala-Ser-Chg-Gln-Ser-Leu-OH) was purchased from Cellmano, China. (1-[Bis(dimethylamino)methylene]-1H-1,2,3triazolo[4,5-b]pyridinium 3-oxid hexafluorophosphate (HATU), N,Ndiisopropylethylamine (DIPEA), doxorubicin hydrochloride (Dox), acetonitrile, ammonium acetate, HEPES, sodium chloride, citric acid, poly-D-lysine, resazurin sodium salt, and cholesteryl hemisuccinate (CHEMS) were all acquired from Sigma Aldrich, UK. Dimethylformamide (DMF) was purchased from Fisher Scientific, UK. 1,2-dioleoyl-snglycero-3-phosphoethanolamine (18:1 ( $\triangle 9$-Cis) PE, DOPE) was obtained from Avanti Polar Lipids (USA).

\section{Synthesis of Glutaryl-Hyp-Ala-Ser-Chg-GIn-Ser-Leu-Dox (Dox-PSA)}

To a solution of PSA-cleavable heptapeptide $(0.0368 \mathrm{mmol}, 48.00 \mathrm{mg})$ in DMF $(5 \mathrm{~mL})$, HATU $(0.0368 \mathrm{mmol}, 13.99 \mathrm{mg})$ was added. The solution was stirred for 30 minutes at RT followed by the addition of doxorubicin hydrochloride $(0.0735 \mathrm{mmol}, 42.60 \mathrm{mg})$. The 
$\mathrm{pH}$ of the reaction was neutralised by the addition of anhydrous DIPEA ( $80 \mu \mathrm{L}, 0.459$ mmol) by using $\mathrm{pH}$ paper. The reaction was stirred for 18 hours at RT under nitrogen whilst covered in foil to prevent any photodegradation of doxorubicin. After completion, the reaction was concentrated under vacuum to dark red oil. The crude product was dissolved in $50 \mathrm{~mL}$ of a buffer consisting of $0.1 \%(\mathrm{w} / \mathrm{v})$ ammonium acetate in water $(80 \%$, buffer A) and acetonitrile (20\%, buffer B).

\section{Purification of Dox-PSA}

The solution of the crude Dox-PSA was passed through a $0.2 \mu \mathrm{m}$ PES filter (Millipore ${ }^{\mathrm{TM}}$ ) before being purified by preparative RP-HPLC (Gilson) on a C18 150 x $4.6 \mathrm{~mm}$ column (Agilent). A step gradient consisting of buffers A and B was optimised as follows: $35 \%$ $\mathrm{B}$ in 2 minutes, $35-80 \% \mathrm{~B}$ in 4 minutes, $80 \% \mathrm{~B}$ in 1 minute, and $80-35 \% \mathrm{~B}$ in 2 minutes. Injection volumes of $4 \mathrm{~mL}$ were performed per run. A flow rate of $20 \mathrm{~mL} / \mathrm{min}$ was used to elute the product, which was detected using a UV detector at $214 \mathrm{~nm}$. Pure fractions were pooled and acetonitrile was removed prior to freeze drying. The dried product was re-dissolved in $0.1 \%$ ammonium acetate, aliquoted, and freeze dried again. The dried powder was stored at $-80{ }^{\circ} \mathrm{C}$. Mass spectrometry (MALDI-TOF) was performed to confirm the identity of the chromatographic peaks. Purity of the product (31 mg, 23\% reaction yield) was checked by analytical HPLC and determined to be $>98 \%$.

\section{Preparation of DOPE:CHEMS-Dox-PSA liposomes}

Phospholipids were dissolved in chloroform: methanol (4:1 v/v) mixture. DOPE:CHEMS (3:2 molar ratio) liposomes were prepared by the lipid film hydration method, as described previously ${ }^{26}$. Briefly, the organic solvents were evaporated under vacuum at $37^{\circ} \mathrm{C}$ for 1 hour using a rotary evaporator (BÜCHI, Labortechnik AG), then flushed with $\mathrm{N}_{2}$ stream to remove any residual traces of organic solvent. Dox-PSA was loaded into DOPE:CHEMS by passive loading. Briefly, the dried lipid film was hydrated at room temperature (RT) with of Dox-PSA in HEPES pH 8.0 by vigorous pipetting to ensure complete hydration, achieve a final lipid and Dox-PSA concentration of $5 \mathrm{mM}$ and 0.25 $\mathrm{mM}$, respectively. Next, liposomes were downsized by extrusion through $0.8 \mu \mathrm{m}$ ( 7 cycles), $0.2 \mu \mathrm{m}$ (17 cycles) and $0.1 \mu \mathrm{m}$ (13 cycles) polycarbonate filters using a miniExtruder (Avanti Polar Lipids). Liposomes were then flushed with $\mathrm{N}_{2}$ and allowed to anneal overnight at RT, to maximise prodrug encapsulation efficiency. Unencapsulated 
Dox-PSA was removed by passing the liposome suspension through a $\mathrm{qEV}$ size exclusion column (IZON Science Europe Ltd). Dox-PSA encapsulation efficiency (EE, \%) was determined by comparing prodrug fluorescence intensity before and after purification, following the equation below:

$$
\text { Dox-PSAEE }(\%)=\frac{I(t) \text { after purification }}{I(t) \text { before purification }} * 100
$$

where $I(t)$ is the fluorescence intensity of liposome suspension after liposome lysis with $1 \%(w / v)$ Triton X-100. Liposomes were concentrated to the initial concentration.

\section{Dynamic Light Scattering (DLS)}

Measurement of the hydrodynamic diameter and zeta potential $(\zeta$-potential) of the prepared formulations were performed using DLS with a Malvern Zetasizer Nano ZS (Malvern). Disposable polystyrene cells and disposable plain folded capillary Zeta cells (Malvern) were used. Liposome suspensions were diluted in $0.2 \mu \mathrm{m}$ filtered deionized water at ratios of 1:100 for size measurements and 1:10 for ZP measurements. All measurements were performed at $25^{\circ} \mathrm{C}$. The hydrodynamic size was presented as the average value of 15 runs with triplicate measurements within each run, while $\zeta$-potential measurements were performed in quintuplicate.

\section{Cryo-transmission electron microscopy (Cryo-TEM)}

Samples for Cryo-electron microscopy were prepared by pipetting $3 \mathrm{ul}$ of solution onto a holy carbon coated grid (Quantifoil 3.5/1). The grid was blotted and vitrified in ethane in a Vitrobot (FEI, The Netherlands). Grids were observed in a FEI T20 electron microscope operating at $200 \mathrm{keV}$ with a Gatan model 626 cryo-stage. Images were recorded under low-dose conditions with a slow scan CCD camera

\section{Freeze fracture electron microscopy}

For freeze-fracture electron microscopy, Dox-PSA-loaded and empty DOPE:CHEMS liposomes were initially cryoprotected by incubation in $30 \%$ glycerol in $0.1 \mathrm{M}$ cacodylate buffer for 60 minutes at $4^{\circ} \mathrm{C}$. Subsequently, $1.5 \mu 1$ of the sample was placed on the copper holder and fast-frozen by immersion into Freon, cooled by liquid nitrogen $\left(-196^{\circ} \mathrm{C}\right)$. Frozen samples were immediately transferred to freeze-fracture device (BALZERS, BAF200), fractured (knife temperature was $-150^{\circ} \mathrm{C}$ ), shadowed with platinum (nominal angle $45^{\circ}$ ) and further strengthened by carbon (nominal angle $90^{\circ}$ ). Replicas were 
transferred to room temperature and cleaned in sodium hypochlorite solution. Cleaned replicas were picked on copper microscopic grids and were examined with transmission electron microscope (Philips, CM100) running at $80 \mathrm{kV}$.

\section{Cell culture}

LNCaP (CRL-1740 $\left.{ }^{\mathrm{TM}}\right)$ and PC3 (CRL-1435 ${ }^{\mathrm{TM}}$ ) cells were obtained from American Type Culture Collection (ATCC $\AA$, USA). LNCaP-derived C4-2B cells were purchased from (MD Anderson Cancer Center, Texas, USA). PC cells were cultured in Advanced RPMI 1640 (Invitrogen Gibco ${ }^{\circledR}$ Life Technologies), supplemented with 10\% FBS, $50 \mathrm{U} / \mathrm{ml}$ penicillin, $50 \mu \mathrm{g} / \mathrm{ml}$ streptomycin and 1\% L-glutamine and maintained in a humidified chamber at $37^{\circ} \mathrm{C}$ and $5 \% \mathrm{CO}_{2}$. $\mathrm{C} 4-2 \mathrm{~B}$ cells were routinely grown in $75 \mathrm{~cm}^{2}$ canted-neck CellBIND ${ }^{\circledR}$ tissue culture flasks (Corning) to improve cell adherence, and passaged twice a week using $0.05 \%$ Trypsin/ EDTA when reaching $80 \%$ confluence in order to maintain exponential growth.

\section{Quantification of free PSA}

$1 \times 10^{5}$ cells were seeded in 6 -well culture plates and 24,48 and $72 \mathrm{~h}$ after incubation at $37^{\circ} \mathrm{C} / 5 \% \mathrm{CO}_{2}$, cell lysates and supernatants were collected. The presence of free PSA in LNCaP and $\mathrm{C} 4-2 \mathrm{~B}$ cell lysates and supernatants was quantitatively measured using the Free PSA Human SimpleStep ELISA ${ }^{\mathrm{TM}}$ Kit (Abcam) according to the manufacturer's instructions.

\section{Uptake studies by Confocal Laser Scanning Microscopy (CLSM)}

\section{Monolayers}

C4-2B cells $\left(6 \times 10^{4}\right)$ were seeded onto $13 \mathrm{~mm}$ glass coverslips (Fisher Scientific) precoated with poly-D-lysine in 24-well plates (Sardstedt). After 48 hours, cells were incubated in complete media containing $5 \mu \mathrm{M}$ of free Dox-PSA or DOPE:CHEMS-DoxPSA liposomes. At different time points, cells were washed twice with $500 \mu \mathrm{L}$ of PBS and fixed with $0.5 \mathrm{~mL}$ of 4\% (v/v) paraformaldehyde in PBS (RT for $15 \mathrm{~min}$ in the dark). Cells were washed twice with PBS to remove fixative and then incubated with $1 \mu \mathrm{g} / \mathrm{mL}$ of Hoechst 33342 (ThermoFisher) for $10 \mathrm{~min}$ to allow nuclei visualisation. Following counterstaining, the coverslips were mounted on glass slides (Scientific Laboratory Supplies) using ProLong ${ }^{\text {TM }}$ Gold Antifade mounting media (Invitrogen Life Technologies). Confocal images were acquired with a Leica SP8 microscope (Leica 
microsystems) using a 63x oil immersion objective (numerical aperture 1.4) and $405 \mathrm{~nm}$ and $488 \mathrm{~nm}$ laser detectors in the hybrid (HyD) mode. Image analysis was performed using the LasX software (Leica microsystems).

\section{Spheroids}

$200 \mu \mathrm{L}$ of C4-2B cells $\left(5 \times 10^{3}\right)$ were seeded in 96-well clear round bottom Ultra-Low Attachment plates (Corning $\left.{ }^{\circledR}\right)$. Media was partially replenished every two days by withdrawing $100 \mu \mathrm{L}$ of spent media and replacing with equivolume of fresh complete media. On day 3, $5 \mu \mathrm{M}$ of free Dox-PSA or DOPE:CHEMS-Dox-PSA liposomes were diluted in $30 \mu \mathrm{L}$ of media and added to the cells. At 48 hours post-incubation, spheroids were thoroughly washed with phenol red-free media and immediately imaged with a Leica SP8 microscope (Leica microsystems) using a 10x dry objective (numerical aperture 0.3 ) and $488 \mathrm{~nm}$ laser detector in the hybrid (HyD) mode. Sequential Z-stack images were acquired from the top to the centre of the spheroids. Image analysis was performed using the LasX software (Leica microsystems).

\section{Cell viability studies in Prostate Cancer Monolayers}

\section{Resazurin assay}

C4-2B cells $\left(1 \times 10^{4}\right)$ were seeded in poly-D-lysine coated 96-well culture plates (Sardstedt) in complete media. After 48 hours, different concentrations of free Dox-PSA or DOPE:CHEMS-Dox-PSA liposomes were diluted in $30 \mu \mathrm{L}$ of media and added to the cells. Media was not replenished to ensure sufficient PSA concentrations. At 48, 72 and 96 hours post-incubation, a resazurin cell viability assay was performed, which is based on the mitochondrial metabolic activity of live cells. Resazurin reagent was prepared as described elsewhere ${ }^{27}$. Briefly, cells were incubated with $0.01 \mathrm{mg} / \mathrm{mL}$ resazurin solution for $4 \mathrm{~h}$. After incubation, fluorescence $\left(\lambda_{\mathrm{ex}}=544 \mathrm{~nm}, \lambda_{\mathrm{em}}=590 \mathrm{~nm}\right)$ was read using an automated FLUOstar Omega (BMG Labtech, UK) plate reader. The results were expressed as the percentage of cell viability (mean $\pm \mathrm{SEM}$ ) and normalised to vehicletreated cells.

\section{Real-Time Cell Analyser (RTCA)}

Experiments carried out in the RTCA DP xCelligence system (ACEA Biosciences, USA) were performed following manufacturer's instructions. A background impedance measurement was done by adding $0.1 \mathrm{~mL}$ of complete media to each well of a poly-Dlysine coated 16-well E-plate (ACEA Biosciences, USA). C4-2B cells were seeded at 1 
x $10^{4}$ cells per well in $0.1 \mathrm{~mL}$ of complete media and allowed to equilibrate for 30 minutes at RT. The plates were then placed in the RTCA DP system inside a humidified chamber at $37^{\circ} \mathrm{C} / 5 \% \mathrm{CO}_{2}$. Cell proliferation was recorded every 15 minutes over a period of 48 hours at which point the experiment was paused, $30 \mu \mathrm{L}$ of media were removed from each well, and cells were treated with $30 \mu \mathrm{L}$ of different concentrations of Dox-PSA or DOPE:CHEMS-Dox-PSA liposomes. Immediately after compound addition, the plates were returned to the RTCA DP system. The assay was then resumed, and measurements were performed every 15 minutes for a period of 72 hours. Experiments were performed in duplicate and cell index was normalised to the time point prior addition of the compounds.

\section{Cell viability in 3D Prostate Cancer Spheroids}

$200 \mu \mathrm{L}$ of C4-2B cells were seeded at a seeding density of $5 \times 10^{3}$ cells/well in 96-well plates pre-coated with $1 \%(\mathrm{w} / \mathrm{v})$ agarose (Sigma Aldrich) using the liquid overlay technique ${ }^{28}$. Media was partially replenished every two days by withdrawing $100 \mu \mathrm{L}$ of spent media and replacing with equivolume of fresh complete media. On day 3 , different concentrations of free Dox-PSA or DOPE:CHEMS-Dox-PSA liposomes were diluted in $30 \mu \mathrm{L}$ of media and added to the cells. At 48-96 hours post-incubation, $100 \mu \mathrm{L}$ of mediacontaining drugs were removed and equal volume of a $10 \mathrm{mM}$ EDTA disodium salt solution was added to yield $5 \mathrm{mM}$ final EDTA concentration. After a 30 minutes incubation at $37^{\circ} \mathrm{C} / 5 \% \mathrm{CO}_{2}$, resazurin was added to a final concentration of $0.01 \mathrm{mg} / \mathrm{mL}$. Spheroids were then incubated overnight at $37^{\circ} \mathrm{C} / 5 \% \mathrm{CO}_{2}$ and the following day, 100 $\mu \mathrm{L}$ of media-containing resazurin were transferred to black plates (Sterilin ${ }^{\mathrm{TM}}$ ) before determining fluorescence. EDTA treatment was used as previously described ${ }^{27}$ with only minor modifications, in order to break the tight junctions on the highly packed C4-2B spheroids, so that resazurin could efficiently penetrate trough the different cells layers.

\section{Results}

\section{Dox-PSA selectively kills PSA-positive cells}

The structure of Dox-PSA is shown in Figure 1A. Mass and purity were confirmed by MALDI-TOF and HPLC analysis, respectively (Figure S1A \& S1B). Selectivity of this prodrug has been previously shown in human PSA-expressing PC cell lines ${ }^{15}$. These studies used LNCaP cells as the PSA-expressing cell line, however, these cells represent 
early stage PC as they display an androgen-sensitive growth and are poorly tumorigenic ${ }^{29-}$ 31 . In the present work, we used androgen-independent C4-2B (PSA-expressing) and PC3 (PSA-non-expressing) cells. C4-2B cell line was selected as it constitutes a better model for $\mathrm{mCRPC}^{32}$. Furthermore, we quantified the intracellular (cell lysates) and extracellular (cell supernatants) levels of free PSA over 72 hours of culture and showed that, although a great fraction of PSA was secreted to the extracellular media, fairly good levels of PSA could also be found intracellularly (Figure 1B). Moreover, C4-2B cells produced significantly higher amounts of free PSA compared to LNCaP cells, suggesting that C42B cells could potentially constitute a more predictive model to study PSA-cleavable prodrugs for mCRPC. Interestingly, similar levels of free PSA can be found in cell lysates and supernatants of C4-2B cells up to 48 hours of culture. However, as the cells are allowed more time to adhere and grow, PSA secretion outweighs the amount of free PSA inside the cells. Nevertheless, after 72 hours, the concentration of secreted free PSA from LNCaP cells was comparable to the intracellular concentration of free PSA in C4-2B cells. We next evaluated the selectivity of Dox-PSA by treating androgen-independent C4-2B and PC3 (negative control) cells with the free prodrug. Interestingly, Dox-PSA was a potent cytotoxic agent towards $\mathrm{C} 4-2 \mathrm{~B}$ cells, whilst minimal toxicity was obtained in PC3 cells (Figure 1C). The calculated IC50 (concentration of prodrug that results in $50 \%$ cell death) for C4-2B cells at 72 hours of incubation was approximately $3.37 \mu \mathrm{M}$, whereas the maximum concentration tested $(10 \mu \mathrm{M})$ was not sufficient to reach this parameter in $\mathrm{PC} 3$ cells. The $\mathrm{IC}_{50}$ reported in the literature for $\mathrm{LNCaP}$ cells incubated for 72 hours with Dox-PSA is $5 \mu \mathrm{M}^{15}$, which is comparable to the one achieved here in C42B cells. Yet, the somewhat lower $\mathrm{IC}_{50}$ obtained for $\mathrm{C} 4-2 \mathrm{~B}$ cells might be attributed to the increased amounts of free PSA in these cells as opposed to LNCaP cells. We have also investigated the intracellular fate of Dox-PSA following uptake by PSA-secreting and non-secreting cells. To that end, cells were incubated with $5 \mu \mathrm{M}$ of Dox-PSA for 24 and 48 hours, after which confocal laser scanning microscopy (CLSM) was performed. Dox-PSA intrinsic fluorescence was used to easily track the prodrug inside the cells. The concentration used was selected as it is near the $\mathrm{IC}_{50}$ and as lower concentrations could not be used due to detection limits. In addition, this concentration was previously used elsewhere ${ }^{33}$. Strikingly, Dox fluorescence signals were detected in the nuclei of C4-2B cells at both time points, whereas in PC3 cells, the prodrug was localised in the cytosol and perinuclear region, with no signal co-localisation in the nuclei (Figure 1D). These results show, for the first time, Dox selective nuclear uptake in PSA-expressing cells, 
suggesting PSA-mediated proteolytic cleavage of Dox-PSA, which resulted in free Dox trafficking to the nucleus. Whether Dox-PSA activation was carried out by extracellularly secreted PSA, resulting in free Dox diffusion across the plasma membrane, or whether it was accomplished intracellularly remains unclear. In efforts to elucidate if Dox-PSA could be activated intracellularly by enzymatically active PSA present in C4-2B cells, we encapsulated it in $\mathrm{pH}$-sensitive liposomes, as will be described below. By using this responsive nanocarrier we want to ensure that: 1) Dox-PSA is "protected" and thus would not be hydrolysed by extracellularly secreted PSA, and 2) the fusogenic nature of these liposomes would ensure a rapid and efficient prodrug release once inside the acidic endosomes $^{23}$, thus making Dox-PSA available for activation by enzymatically active PSA present in C4-2B cells.

\section{Dox-PSA encapsulation and release from $\mathrm{pH}$-sensitive liposomes}

Different drug loading methods were investigated in order to select the one that provided the highest encapsulation efficiency (Figure S2). Passive encapsulation (Figure 2A) rendered significantly higher encapsulation efficiency $(39.4 \pm 10.3 \%)$ than the one achieved with either freeze-thawing $(23.5 \pm 0.96 \%)$ or remote loading $(15.2 \pm 1.2 \%)$ and therefore passive loading was used in further experiments (Figure 2C). Drug incubation time, i.e., the time frame between extrusion and purification was also tested to assess if longer periods could impact on the encapsulation efficiency. Figure 2D shows that the incubation time remarkably influenced the amount of encapsulated Dox-PSA, which indicates that the mechanism behind Dox-PSA loading in DOPE:CHEMS liposomes is a combination of passive and remote loading. In an attempt to further maximise Dox-PSA loading, we varied the initial lipid concentration. We hypothesised that having more liposomes per unit of volume would increase Dox-PSA passive loading in the liposome core. Yet, the latter was not the case as doubling the total lipid concentration from 5 to 10 $\mathrm{mM}$ did not improve encapsulation efficiency (Figure 2E), therefore, all future experiments were performed using $5 \mathrm{mM}$ of total lipid. Importantly, prodrug-loaded DOPE:CHEMS liposomes displayed sizes compatible with the EPR effect $(157.8 \pm 0.9$ $\mathrm{nm})$. Furthermore, Dox-PSA encapsulation did not result in an increase in liposome size nor did it alter the $\zeta$-potential of these negatively charged liposomes (Table 1), likely indicating that the prodrug resides in the liposome core rather than being adsorbed to the liposome surface. Next, to elucidate liposome structure, the prodrug-loaded liposomes 
were examined using cryogenic-transmission electron microscopy (cryo-TEM) and freeze-fracture electron microscopy (FF-EM). Both techniques showed that indeed the size and morphology of Dox-PSA-loaded liposomes resembled that of their empty counterparts (Figure 3). Furthermore, the appearance of large prodrug crystals inside the liposomes was not evident, suggesting the presence of the prodrug in its soluble form.

Table 1 | Physiochemical characterisation of the optimised DOPE:CHEMS-DoxPSA liposomes

\begin{tabular}{cccc}
\hline Formulation & Z-ave (d.nm) & PdI & $\begin{array}{c}\zeta \text {-potential } \\
(\mathbf{m V})\end{array}$ \\
\hline Empty & $158.1 \pm 1.3$ & $0.073 \pm 0.02$ & $-54.9 \pm 2.53$ \\
Z-ave: hydrodynamic size, PdI: polydispersity index, $\zeta$-potential: surface charge
\end{tabular}

Next, we evaluated the prodrug release from DOPE:CHEMS liposomes at $37^{\circ} \mathrm{C}$ and $\mathrm{pH}$ 5.5 and 7.4. As expected, higher Dox-PSA was released under acidic $\mathrm{pH}$ than physiological pH (Figure S3 A). It is important to highlight that the release studies were carried out in PBS, and might not directly represent the "real" in vitro and in vivo situation, where longer incubation in a more complex medium and cellular uptake could result in higher release and cell toxicity.

\section{Dox-PSA can be released from pH-sensitive liposomes and intracellularly activated in $\mathbf{C} 4-2 B$ cells}

Following the successful encapsulation of Dox-PSA into DOPE:CHEMS liposomes, we then assessed the intracellular fate of either the free Dox-PSA or DOPE:CHEMS-DoxPSA in C4-2B cells at an equivalent dose of $5 \mu \mathrm{M}$ Dox-PSA. Once again, the fluorescence properties of the prodrug and the fact that, unlike Dox, its fluorescence is not quenched when encapsulated in liposomes (data not shown), allowed us to easily track the subcellular localisation of the prodrug/liposomes by CLSM. As depicted in Figure 4A, liposomes were more rapidly accumulated intracellularly compared to the free prodrug. These results are likely due to the fusogenic properties of DOPE lipid, which has a poorly hydrated polar head group, resulting in high affinity for the cellular membrane ${ }^{34,35}$. Nevertheless, after 24 hours of incubation at $37^{\circ} \mathrm{C}$, the liposomal Dox-PSA was still 
distributed throughout the cytosol of the cells, whereas Dox nuclear uptake was evident in the case of the free prodrug (Figure 4B). These observations are not surprising if we take into account that encapsulation of Dox-PSA inside a delivery system represents an additional barrier to PSA-mediated hydrolysis. Yet, longer incubation time with DOPE:CHEMS-Dox-PSA liposomes allowed the prodrug to be activated and Dox translocated from the cytosol to the nuclei of those cells. It is noteworthy to highlight the relevance of these results as they clearly support the possibility of intracellular activation of Dox-PSA in C4-2B cells, following internalisation, since intact Dox-PSA did not traffic to the nucleus (Figure 1D). Moreover, by encapsulating Dox-PSA inside DOPE:CHEMS liposomes we ruled out any potential prodrug cleavage in the extracellular space. Interestingly, Dox nuclear localisation in cells incubated with the free and encapsulated Dox-PSA demonstrates that there were enough amounts of enzymatically active PSA inside C4-2B cells to cleave Dox from the PSA peptide. Furthermore, the presence of nuclear fluorescent signals shows that DOPE:CHEMS liposomes successfully released the prodrug that was followed by proteolysis by intracellular PSA. These data are exciting as they open up the possibility of using a delivery system of Dox-PSA, which could protect the prodrug while in the systemic circulation, thus bypassing any potential non-specific hydrolysis.

\section{DOPE:CHEMS-Dox-PSA liposomes induce toxicity in C4-2B monolayers}

To explore if the intracellular activation of Dox-PSA could result in significant cell toxicity, a resazurin assay was used to determine the viability of C4-2B cells treated with different doses of either free Dox-PSA or DOPE:CHEMS-Dox-PSA liposomes. Moreover, to ensure that any observed toxicities were a result of Dox-PSA release from the liposomes, followed by its enzymatic activation, and not due to the carrier itself, the toxicity of empty DOPE:CHEMS was assessed, where no toxicity was observed up to 96 hours of incubation (Figure 5A). Conversely, dose- and time-dependent reductions in the viability of $\mathrm{C} 4-2 \mathrm{~B}$ cells treated with both the free prodrug and the $\mathrm{pH}$-sensitive liposomes were observed. Furthermore, DOPE:CHEMS-Dox-PSA liposomes displayed toxicity levels similar to those achieved with the free Dox-PSA (Figure 5B \& 5C), indicating successful liposomal release of Dox-PSA, and enzymatic activation. More interestingly, C4-2B cells treated with conventional DPPC:Chol:DSPE-PEG2000-Dox-PSA liposomes showed no toxicity up to 96 hours (Figure S3 B), indicating no significant prodrug leakage from these liposomes. These findings, suggest the need of a stimuli to trigger 
Dox-PSA release and support the selective release from DOPE:CHEMS liposomes under acidic environment (Figure S3 A).

It is worth mentioning that at some concentrations and time points, DOPE:CHEMS-DoxPSA liposomes were slightly less toxic than the free Dox-PSA. Such observations could be explained by either an incomplete prodrug release from the liposomes at a certain time point, or by the added barrier to prodrug activation that the liposomes represent, which might delay the toxicity profile in comparison to the free prodrug. Furthermore, similar findings were obtained using the label-free Real Time Cell Analysis (RTCA) (Figure 5DF). Our RTCA results, showed that the $\mathrm{IC}_{50}$ values for the free Dox-PSA and DOPE:CHEMS-Dox-PSA liposomes were comparable at 24 hours, but over time the free prodrug was slightly more toxic. Importantly, at 24 hours of incubation, $10 \mu \mathrm{M}$ of DoxPSA was more toxic in the liposomal form $\left(\mathrm{I}_{\max }=28.52 \pm 0.01 \%\right)$ than as free prodrug $\left(\mathrm{I}_{\max }=42.57 \pm 0.01 \%\right.$ ) (Table 2$)$. Overall, the cell viability data obtained with the endpoint and real-time assays showed that Dox-PSA delivered by DOPE:CHEMS liposomes, although slightly less potent than the free Dox-PSA, preserves the cytotoxic activity.

Table 2 | Dox-PSA and DOPE:CHEMS-Dox-PSA potency and efficacy in C4-2B monolayers, as determined by RTCA.

\begin{tabular}{|c|c|c|c|c|c|c|}
\hline & \multicolumn{3}{|c|}{$\mathrm{IC}_{50} \pm \mathrm{SD}(\boldsymbol{\mu M})$} & \multicolumn{3}{|c|}{$I_{\max } \pm \operatorname{SD}(\%)$} \\
\hline & 24 hours & 48 hours & 72 hours & 24 hours & 48 hours & 72 hours \\
\hline Dox-PSA & $3.91 \pm 1.28$ & $0.31 \pm 0.08$ & $0.14 \pm 0.03$ & $42.57 \pm 1.28$ & $0.00 \pm 0.00$ & $0.45 \pm 0.63$ \\
\hline DOPE:CHEMS-Dox-PSA & $5.51 \pm 0.20$ & $1.45 \pm 0.15^{\mathrm{a}}$ & $0.68 \pm 0.06^{\mathrm{b}}$ & $28.52 \pm 0.01^{\mathrm{c}}$ & $0.13 \pm 0.18$ & $0.00 \pm 0.00$ \\
\hline
\end{tabular}

Statistical significance was determined using an unpaired t-test, showing significant result between DoxPSA and DOPE:CHEMS-Dox-PSA. ${ }^{\mathrm{a}} *(\mathrm{p}<0.05),{ }^{\mathrm{b}} * *(\mathrm{p}<0.01)$, and ${ }^{\mathrm{c}} * *(\mathrm{p}<0.01)$.

\section{DOPE:CHEMS-Dox-PSA liposomes are therapeutically more effective than the free}

\section{Dox-PSA in C4-2B spheroids}

Given the promising results obtained in C4-2B monolayers, we decided to assess the efficacy of prodrug-loaded liposomes in C4-2B spheroids, which more realistically replicate the in vivo environment. $\mathrm{C} 4-2 \mathrm{~B}$ cells growing on a non-adherent agarose matrix rapidly produced highly compact spheroids which developed a visible hypoxic core. Tumour spheroids were treated with either free or encapsulated Dox-PSA for different 
time periods. Notably, treatment of C4-2B spheroids with DOPE:CHEMS-Dox-PSA liposomes induced a very significant reduction in the viability in contrast to the observed for the free Dox-PSA (Figure 6A-C). These results suggest that Dox-PSA encapsulation in $\mathrm{pH}$-sensitive liposomes contributes to its enhanced penetration across the spheroid cell layers. Furthermore, toxicity of free Dox-PSA was lower in spheroids compared to monolayers, which was not time- or concentration-dependent. Such observation could either indicate that spheroids do not produce sufficient amounts of enzymatically active PSA, or that the free Dox-PSA does not penetrate deeply through the inner layers of the spheroids, as suggested by the absence of toxicity at 48 hours post-incubation (Figure 6A). Interestingly, when Dox-PSA was encapsulated in $\mathrm{pH}$-sensitive liposomes, toxicity levels closely resembled those achieved in monolayers, which excluded the possibility of spheroids not producing enough enzymatically active PSA. Furthermore, the enhanced penetration of the prodrug using the fusogenic, $\mathrm{pH}$-sensitive DOPE-CHEMS liposomes was confirmed using CLSM (Figure 6D). It was clear that free Dox-PSA was located mainly at the peripheral cell layers of the tumour spheroid, which might justify the minimal toxicity obtained with free Dox-PSA at 48 hours. Conversely, the liposomal Dox-PSA penetrated deeper within the spheroids, resulting in higher cell toxicity.

\section{Discussion}

Dox-PSA was developed to improve the therapeutic index of Dox by exploiting PSA relative specificity to the prostate tissue. Previous studies with this prodrug have shown that it was a selective anticancer agent against PSA-secreting cells. In addition, Dox-PSA was approximately 15 -fold more effective in reducing tumour weight compared to Dox in nude mice bearing LNCaP tumours ${ }^{15}$. Furthermore, selectivity studies with Dox-PSAtreated mice, have shown that 2.5 -fold more Dox molar equivalents accumulated in tumour whereas substantially lower concentrations were found in heart tissue in comparison with the situation where Dox was administered alone ${ }^{14}$. Despite the initially promising results, Dox-PSA did not advance beyond phase I clinical trials. The reasons behind this were likely toxicity concerns as it was shown that in four different species of laboratory animals there was approximately 30-40\% non-PSA-mediated prodrug conversion into the active metabolite Dox ${ }^{13,14}$. Aiming at increasing PSA specificity, Aloysius et al. modified the initial peptide sequence, Glutaryl-Hyp-Ala-Ser-Chg-Gln, by introducing modifications at position $\mathrm{P}_{5}$, which improved PSA hydrolysis rate and 
decreased non-PSA-specific cleavage ${ }^{13}$. On the other hand, a safer therapeutic approach based on Dox-PSA encapsulation in a nanocarrier could potentially circumvent the nonspecific activation as the prodrug would be delivered to the tumour cells inside a "shield". Several studies, have used a wide range of liposomes to efficiently deliver a variety of prodrugs, including a number of anticancer agents ${ }^{36,37}$, phospholipid prodrugs ${ }^{38,39}$, antiinflammatory prodrugs ${ }^{40}$ and $\beta$-blockers prodrugs ${ }^{41}$. Interestingly, previous reports with PSA-cleavable prodrugs have relied on the enzymatically active extracellular PSA to activate the prodrug ${ }^{13,15,20,42,43}$. As a proof-of-concept, in this work we used pH-sensitive liposomes composed of DOPE:CHEMS to ensure a fast and efficient Dox-PSA release inside the acidified endosomes, which is essential for prodrug activation. Interestingly, and for the first time, we have provided evidence for the intracellular activation of DoxPSA prodrug following its encapsulation in DOPE:CHEMS liposomes, which did not hinder its cytotoxic activity, but, indeed, improved its activity in 3D PC models.

Here, C4-2B cells were used as a model for mCRPC and we have shown by ELISA that these cells secrete high levels of free PSA in a time-dependent fashion and that, more importantly, higher intra and extracellular concentrations of free PSA were found in C42B cells in comparison to the LNCaP counterparts (Figure 1B), that have been consistently used to assess the selectivity of PSA-cleavable prodrugs ${ }^{12,15,44}$. These observations are relevant as they show that $\mathrm{C} 4-2 \mathrm{~B}$ cells produce high amounts of free PSA, which is not exclusively secreted, but also present inside the cells. The reliance on extracellular cleavage in previous studies with PSA-cleavable prodrugs is based on several factors. Firstly, it is known that divalent ions, specifically zinc, inhibit PSA proteolytic activity ${ }^{20,45}$. However, malignant human prostate epithelial cells contain decreased levels of zinc in comparison with their normal counterparts as a consequence of dysregulation of a number of zinc transporters in PC $21,22,46$. Secondly, it was reported that PSA enzyme is converted into the enzymatically active form upon extracellular removal of a 7 amino acid pro-sequence ${ }^{47}$ and thus, any PSA present intracellularly would be catalytically inactive. However, a previous study has shown that PSA is imported to the nuclei of both $\mathrm{LNCaP}$ and $\mathrm{C} 4-2 \mathrm{~B}$ cells and proposed two models to justify this finding: 1) full length PSA is extracellularly activated and then imported into the nucleus, or 2) PSA is cleaved intracellularly by unknown proteases and then translocated to the nucleus ${ }^{48}$. Independently of whether PSA is converted into the catalytically active form outside the cells and then imported back in, or whether its activation can also be 
accomplished intracellularly, we believe that C4-2B cells might produce enzymatically active PSA that could be explored for the intracellular activation of a Dox-PSA nanomedicine. We have shown that the in-house synthesised Dox-PSA prodrug is selectively toxic towards PSA-expressing C4-2B cells, whereas PSA-non-expressing PC3 cells displayed only minor toxicity (Figure 1C). These data correlate well with the toxicity results reported in the literature using the same prodrug in $\mathrm{LNCaP}$ and PC3 cells $^{15}$. Our selectivity results were further supported by confocal imaging, where cleaved Dox was localised in the nuclei of C4-2B cells at 24 and 48 hours of incubation, but not in the nuclei of PC3 cells, as Dox-PSA fluorescent signal was restricted to the cytosol and perinuclear region (Figure 1D). This intracellular activation of Dox-PSA was further confirmed by encapsulating Dox-PSA inside DOPE:CHEMS liposomes to eliminate any extracellular activation. Thus, any nuclear signals would come from Dox-PSA cleavage by intracellular enzymatically active PSA.

Liposomes are known to efficiently encapsulate a wide range of therapeutics ${ }^{49}$. In the present study, we were able to encapsulate approximately $39.4 \pm 10.3 \%$ Dox-PSA in DOPE:CHEMS liposomes by means of passive encapsulation, (Figure 2C) which was lower than a previous report, using the positively charged 25 natural amino acid peptide Print $3 G^{50}$. The higher entrapment efficiency attained with Print $3 G$ might be due to its higher hydrophilicity and its electrostatic interaction with negatively charged liposomes. Interestingly, our results confirmed that Dox-PSA was entrapped inside the liposomes, since minimum changes in liposomes size, surface charge, and morphology were observed (Table 1 \& Figure 3, respectively). Following a successful Dox-PSA encapsulation, cargo release and endosomal escape are of paramount importance to avoid prodrug degradation as a consequence of the low $\mathrm{pH}$ and enzymatic cocktail present in the endolysosomal system ${ }^{51,52}$, and to ensure Dox-PSA delivery to the cytosol, where it can then be metabolised into Dox. Here, resides the beauty of the $\mathrm{pH}$-sensitive liposomes, which are tailored-made for endosomal escape ${ }^{51,53}$. Using CLSM, we have shown that at early time points, despite their high negative surface charge, they accumulated faster inside C4-2B cells compared to the free Dox-PSA (Figure 4A). Although in the present work the endocytic mechanisms were not investigated, we speculate that the difference in the extent of internalisation between the free prodrug and the $\mathrm{pH}$-sensitive liposomes could rely on the involvement of distinct uptake modalities and/or kinetics of intracellular processing. There is compelling evidence showing that liposome/nanoparticle uptake is 
influenced by a multitude of parameters, including nanocarrier size ${ }^{52,54}$, surface charge $^{55,56}$ and hydrophobicity ${ }^{56,57}$, lipid composition ${ }^{58,59}$, shape $e^{53,56}$ and cell type ${ }^{53,54}$. Based on the available literature, we hypothesise that both the free and encapsulated DoxPSA are taken up by PC cells via caveolae-mediated endocytosis (CME), in contrast to free Dox which is known to freely diffuse across the plasma membrane and readily localise in the nucleus ${ }^{60}$. Indeed, this endocytic pathway had previously been reported for pH-sensitive liposomes ${ }^{23,61}$. Furthermore, the observation that Dox-PSA fluorescence was initially localised in the cytosol of C4-2B cells and gradually appeared in the nuclei in a time-dependent manner, indicates that the prodrug does not enter the nucleus until it is cleaved into free Dox ${ }^{62}$, since the latter was not observed in PSA-negative cells (Figure 1D). As expected, Dox-PSA encapsulation in DOPE:CHEMS liposomes resulted in a delayed nuclear uptake, since the prodrug must first be released from the carrier ${ }^{58}$ prior its activation. Remarkably, longer incubation time enabled released Dox to travel to the nuclei of C4-2B cells (Figure 4A \& 4B), which is an indication of efficient prodrug release and subsequent activation by enzymatically active PSA present in these cells. These results were further confirmed since relatively comparable cytotoxicity of free and encapsulated Dox-PSA in C4-2B was observed. It is worth mentioning that some differences in the IC50 values were observed between resazurin and RTCA, however, such discrepancies were reported previously ${ }^{63}$. Lower viabilities were reported using RTCA compared to resazurin, since the former measures cell impedance which is highly sensitive to any stress that can potentially cause an alteration in the cellular morphology and/or adherence strength, which enabled us to detect changes, 24 hours earlier than resazurin assay (48 hours). Furthermore, metabolism-based assays such as resazurin assay have been reported to overestimate cell viability in comparison with nuclei-counting approach ${ }^{63}$. This could explain the differences in the IC50 of Dox-PSA determined by resazurin (Figure 5B\& 5C) and RTCA (Figure 5D-F). Nevertheless, despite these differences, our findings consistently showed that DOPE:CHEMS-Dox-PSA showed high cytotoxicity, further supporting Dox-PSA release from the liposomes and its delivery into the cytoplasm.

Although 2D models constitute an easy and practical solution in the preclinical validation of drugs, they are far too simplistic to replicate the complexity of the pathophysiology of tumours. To overcome the bottlenecks associated with cell monolayers, 3D models are being increasingly used as they more realistically recapitulate the in vivo tumour biology, 
in respect to cell-cell interactions, the presence of extracellular matrix and cancer stem cells (CSC) properties, oxygen, $\mathrm{pH}$ and nutrient gradients, hypoxic regions and necrotic core, and gene expression profiles ${ }^{64,65}$. It has been reported that PC spheres are enriched in CSC in comparison with adherent cells. This is relevant as CSC are implicated in tumour initiation and progression, metastatic disease, and chemotherapy resistance ${ }^{66}$. In an attempt to evaluate the relevance of the DOPE:CHEMS-Dox-PSA liposomes in the therapy of advanced PC, we then assessed their toxicity in C4-2B spheroids. Unlike the results obtained in monolayers, incubation of $\mathrm{C} 4-2 \mathrm{~B}$ spheroids with the $\mathrm{pH}$-sensitive liposomes resulted in significantly augmented toxicity in comparison to the free prodrug (Figure 6A-C). Such discrepancies could be justified by an enhanced penetration of these fusogenic liposomes ${ }^{67}$ over the free prodrug, as confirmed by confocal imaging, where high fluorescent signals were detected at deeper levels in the treated spheroids (Figure 6D).

All in all, we have sucessfully demonstrated that Dox-PSA prodrug can be intracellularly activated in C4-2B cells. Furthermore, uptake and cytotoxicity studies showed that: 1) DOPE:CHEMS liposomes can efficiently deliver Dox-PSA to C4-2B cells, 2) Dox-PSA can be efficiently released from these $\mathrm{pH}$-sensitive liposomes, 3 ) the released prodrug can be activated into Dox, followed by translocation to the nuclei of PSA-expressing cells, and 4) DOPE:CHEMS-Dox-PSA liposomes were able to retain prodrug activity in 2D cultures, whilst they were more toxic than the free Dox-PSA in 3D PC models. Overall, we propose that revisiting this "forgotten" prodrug and combining it with a nanomedicine approach can be a smart strategy to overcome "off-target" prodrug activation. Further in vivo studies are needed to validate the use of such a nanomedicine in the treatment of mCRPC, however, these nanomedicines should be engineered to exhibit relatively long blood circulation to passively target tumours, while promptly releasing the encapsulated prodrug in response to external stimuli (e.g. $\mathrm{pH}$ or temperature), thus enabling its intracellular activation.

\section{References}

1. Litwin, M. S. \& Tan, H.-J. The Diagnosis and Treatment of Prostate Cancer. JAMA 317, 2532 (2017). 
2. Sumanasuriya, S. \& De Bono, J. Treatment of Advanced Prostate Cancer-A Review of Current Therapies and Future Promise. Cold Spring Harb. Perspect. Med. 8, a030635 (2018).

3. Rautio, J. et al. Prodrugs: Design and clinical applications. Nat. Rev. Drug Discov. 7, 255-270 (2008).

4. Choi, K. Y., Swierczewska, M., Lee, S. \& Chen, X. Protease-Activated Drug Development. Theranostics 2, 156-179 (2012).

5. Rautio, J., Meanwell, N. A., Di, L. \& Hageman, M. J. The expanding role of prodrugs in contemporary drug design and development. Nat. Rev. Drug Discov. (2018). doi:10.1038/nrd.2018.46

6. Yang, F., Teves, S. S., Kemp, C. J. \& Henikoff, S. Doxorubicin, DNA torsion, and chromatin dynamics. Biochim. Biophys. Acta - Rev. Cancer 1845, 84-89 (2014).

7. Lyu, Y. L. \& Liu, L. F. in Recent Advances in Cancer Research and Therapy 351-369 (Elsevier, 2012). doi:10.1016/B978-0-12-397833-2.00013-3

8. Lilja, H., Ulmert, D. \& Vickers, A. J. Prostate-specific antigen and prostate cancer: prediction, detection and monitoring. Nat. Rev. Cancer 8, 268-278 (2008).

9. Barve, A., Jin, W. \& Cheng, K. Prostate cancer relevant antigens and enzymes for targeted drug delivery. J. Control. Release 187, 118-132 (2014).

10. Yang, Y., Aloysius, H., Inoyama, D., Chen, Y. \& Hu, L. Enzyme-mediated hydrolytic activation of prodrugs. Acta Pharm. Sin. B 1, 143-159 (2011).

11. Tomao, L. et al. Characterization of the Prostate-Specific Antigen (PSA) Catalytic Mechanism: A Pre-Steady-State and Steady-State Study. PLoS One 9, e102470 (2014).

12. Garsky, V. M. et al. The synthesis of a prodrug of doxorubicin designed to provide reduced systemic toxicity and greater target efficacy. J. Med. Chem. 44, 4216-24 (2001).

13. Aloysius, H. \& Hu, L. Improving the Specificity of the Prostate-Specific Antigen Substrate Glutaryl-Hyp-Ala-Ser-Chg-Gln as a Promoiety. Chem. Biol. Drug Des. 86, 837-848 (2015).

14. Wong, B. K. et al. PSA-specific and non-PSA-specific conversion of a PSAtargeted peptide conjugate of doxorubicin to its active metabolites. Drug Metab. Dispos. 29, 313-318 (2001). 
15. DeFeo-Jones, D. et al. A peptide-doxorubicin 'prodrug' activated by prostatespecific antigen selectively kills prostate tumor cells positive for prostate-specific antigen in vivo. Nat. Med. 6, 1248-1252 (2000).

16. DiPaola, R. S. et al. Characterization of a novel prostate-specific antigenactivated peptide-doxorubicin conjugate in patients with prostate cancer. J. Clin. Oncol. 20, 1874-9 (2002).

17. Yin Wanga, Andrew G. Cheethama, Garren Angacian, Hao Sua, Lisi Xiea, A. \& Cui, H. Peptide-Drug Conjugates as Effective Prodrug Strategies for Targeted Delivery. Adv Drug Deliv Rev. 35, 30-37 (2015).

18. Zaro, J. L. Lipid-Based Drug Carriers for Prodrugs to Enhance Drug Delivery. AAPS J. 17, 83-92 (2015).

19. Giang, I., Boland, E. L. \& Poon, G. M. K. Prodrug Applications for Targeted Cancer Therapy. AAPS J. 16, 899-913 (2014).

20. DeFeo-Jones, D. et al. A prostate-specific antigen (PSA)-activated vinblastine prodrug selectively kills PSA-secreting cells in vivo. Mol. Cancer Ther. 1, 451-9 (2002).

21. Huang, L., Kirschke, C. P. \& Zhang, Y. Decreased intracellular zinc in human tumorigenic prostate epithelial cells: a possible role in prostate cancer progression. Cancer Cell Int. 6, 10 (2006).

22. Ho, E. \& Song, Y. Zinc and prostatic cancer. Curr. Opin. Clin. Nutr. Metab. Care 12, 640-645 (2009).

23. Huth, U. S., Schubert, R. \& Peschka-Süss, R. Investigating the uptake and intracellular fate of $\mathrm{pH}$-sensitive liposomes by flow cytometry and spectral bioimaging. J. Control. Release 110, 490-504 (2006).

24. Lee, Y. \& Thompson, D. H. Stimuli-responsive liposomes for drug delivery. Wiley Interdiscip. Rev. Nanomedicine Nanobiotechnology 9, (2017).

25. Guo, X., MacKay, J. A. \& Szoka, F. C. Mechanism of pH-triggered collapse of phosphatidylethanolamine liposomes stabilized by an ortho ester polyethyleneglycol lipid. Biophys. J. 84, 1784-95 (2003).

26. Van den Bossche, J. et al. Intracellular trafficking and gene expression of $\mathrm{pH}-$ sensitive, artificially enveloped adenoviruses in vitro and in vivo. Biomaterials 32, 3085-3093 (2011).

27. Walzl, A. et al. The Resazurin Reduction Assay Can Distinguish Cytotoxic from Cytostatic Compounds in Spheroid Screening Assays. J. Biomol. Screen. 19, 
1047-59 (2014).

28. Carlsson, J. \& Yuhas, J. M. Liquid-Overlay Culture of Cellular Spheroids. 95, 1$23(1984)$.

29. Russell, P. J. \& Kingsley, E. A. in Prostate Cancer Methods and Protocols 2140 (Humana Press). doi:10.1385/1-59259-372-0:21

30. Dozmorov, M. G. et al. Unique patterns of molecular profiling between human prostate cancer LNCaP and PC-3 cells. Prostate 69, 1077-1090 (2009).

31. Cunningham, D. \& You, Z. In vitro and in vivo model systems used in prostate cancer research. J. Biol. Methods 2, 17 (2015).

32. Spans, L. et al. Comparative Genomic and Transcriptomic Analyses of LNCaP and C4-2B Prostate Cancer Cell Lines. PLoS One 9, e90002 (2014).

33. Ibsen, S. et al. A Novel Doxorubicin Prodrug with Controllable Photolysis Activation for Cancer Chemotherapy. Pharm. Res. 27, 1848-1860 (2010).

34. Karanth, H. \& Murthy, R. S. R. pH-Sensitive liposomes-principle and application in cancer therapy. J. Pharm. Pharmacol. 59, 469-483 (2007).

35. Simões, S., Slepushkin, V., Düzgünes, N. \& Pedroso de Lima, M. C. On the mechanisms of internalization and intracellular delivery mediated by $\mathrm{pH}-$ sensitive liposomes. Biochim. Biophys. Acta - Biomembr. 1515, 23-37 (2001).

36. Ren, G. et al. Docetaxel prodrug liposomes for tumor therapy: characterization, in vitro and in vivo evaluation. Drug Deliv. 23, 1272-1281 (2016).

37. Ling, L. et al. Self-assembled liposomes of dual paclitaxel-phospholipid prodrug for anticancer therapy. Int. J. Pharm. 526, 11-22 (2017).

38. Coert, A., Geelen, J. \& Visser, J. De. The pharmacology and metabolism of testosterone undecanoate (TU), a new orally active androgen. Acta Endocrinol. (Copenh). 79, 789-800 (1975).

39. Hostetler, K. Y. et al. Antiviral activities of oral 1-O-hexadecylpropanediol-3phosphoacyclovir and acyclovir in woodchucks with chronic woodchuck hepatitis virus infection. Antimicrob. Agents Chemother. 44, 1964-1969 (2000).

40. Turjeman, K. \& Barenholz, Y. Liposomal nano-drugs based on amphipathic weak acid steroid prodrugs for treatment of inflammatory diseases. J. Drug Target. 24, 805-820 (2016).

41. Kawakami, S. et al. Sustained ocular delivery of tilisolol to rabbits after topical administration or intravitreal injection of lipophilic prodrug incorporated in liposomes. J. Pharm. Pharmacol. 53, 1157-1161 (2001). 
42. Denmeade, S. R. et al. Prostate-specific antigen-activated thapsigargin prodrug as targeted therapy for prostate cancer. J. Natl. Cancer Inst. 95, 990-1000 (2003).

43. Mhaka, A., Denmeade, S. R., Yao, W., Isaacs, J. T. \& Khan, S. R. A 5fluorodeoxyuridine prodrug as targeted therapy for prostate cancer. Bioorg. Med. Chem. Lett. 12, 2459-61 (2002).

44. Denmeade, S. R. et al. Enzymatic Activation of a Doxorubicin-Peptide Prodrug by Prostate-Specific Antigen Advances in Brief Prodrug by. Cancer Res. 58, 2537-2540 (1998).

45. Malm, J., Hellman, J., Hogg, P. \& Lilja, H. Enzymatic action of prostate-specific antigen (PSA or hK3): Substrate specificity and regulation by Zn2+, a tightbinding inhibitor. Prostate 45, 132-139 (2000).

46. Williams, S. A. et al. Enzymatically active prostate-specific antigen promotes growth of human prostate cancers. Prostate 71, 1595-1607 (2011).

47. Williams, S. A., Xu, Y., De Marzo, A. M., Isaacs, J. T. \& Denmeade, S. R. Prostate-specific antigen (PSA) is activated by KLK2 in prostate cancer ex vivo models and in prostate-targeted PSA/KLK2 double transgenic mice. Prostate 70, 788-96 (2010).

48. Saxena, P. et al. PSA regulates androgen receptor expression in prostate cancer cells. Prostate 72, 769-776 (2012).

49. Sousa, I., Rodrigues, F., Prazeres, H., Lima, R. T. \& Soares, P. Liposomal therapies in oncology: does one size fit all? Cancer Chemother. Pharmacol. 82, 741-755 (2018).

50. Ducat, E. et al. Nuclear delivery of a therapeutic peptide by long circulating $\mathrm{pH}-$ sensitive liposomes: Benefits over classical vesicles. Int. J. Pharm. 420, 319-332 (2011).

51. Kou, L., Sun, J., Zhai, Y. \& He, Z. The endocytosis and intracellular fate of nanomedicines: Implication for rational design. Asian J. Pharm. Sci. 8, 1-10 (2013).

52. Rejman, J., Oberle, V., Zuhorn, I. S. \& Hoekstr, D. Size-dependent internalization of particles via the pathways of clathrin- and caveolae-mediated endocytosis. Biochem. J. 377, 159-169 (2004).

53. Hillaireau, H. \& Couvreur, P. Nanocarriers' entry into the cell: relevance to drug delivery. Cell. Mol. Life Sci. 66, 2873-2896 (2009).

54. Zauner, W., Farrow, N. A. \& Haines, A. M. R. In vitro uptake of polystyrene 
microspheres: effect of particle size, cell line and cell density. J. Control. Release 71, 39-51 (2001).

55. Kang, J. H., Jang, W. Y. \& Ko, Y. T. The Effect of Surface Charges on the Cellular Uptake of Liposomes Investigated by Live Cell Imaging. Pharm. Res. 34, 704-717 (2017).

56. Verma, A. \& Stellacci, F. Effect of surface properties on nanoparticle-cell interactions. Small 6, 12-21 (2010).

57. Ahn, S., Seo, E., Kim, K. \& Lee, S. J. Controlled cellular uptake and drug efficacy of nanotherapeutics. Sci. Rep. 3, 1997 (2013).

58. Mock, J. N., Costyn, L. J., Wilding, S. L., Arnold, R. D. \& Cummings, B. S. Evidence for distinct mechanisms of uptake and antitumor activity of secretory phospholipase A 2 responsive liposome in prostate cancer. Integr. Biol. 5, 172$182(2013)$.

59. Peters, T. et al. Cellular uptake and in vitro antitumor efficacy of composite liposomes for neutron capture therapy. Radiat. Oncol. 10, 1-13 (2015).

60. Dos Santos Câmara, A. L. et al. Acid-sensitive lipidated doxorubicin prodrug entrapped in nanoemulsion impairs lung tumor metastasis in a breast cancer model. Nanomedicine 12, 1751-1765 (2017).

61. Collins, D. S., Findlay, K. \& Harding, C. V. Processing of exogenous liposomeencapsulated antigens in vivo generates class I MHC-restricted T cell responses. J. Immunol. 148, 3336-3341 (1992).

62. Guan, H., Mcguire, M. J., Li, S., Brown, K. C. \& Simmons, T. Peptide-Targeted Polyglutamic Acid Doxorubicin Conjugates for the Treatment of $\alpha \mathrm{v} \beta 6$-Positive Cancers. Bioconjug Chem. 19, 1813-1821 (2008).

63. Single, A., Beetham, H., Telford, B. J., Guilford, P. \& Chen, A. A Comparison of Real-Time and Endpoint Cell Viability Assays for Improved Synthetic Lethal Drug Validation. J. Biomol. Screen. 20, 1286-1293 (2015).

64. Zanoni, M. et al. 3D tumor spheroid models for in vitro therapeutic screening: A systematic approach to enhance the biological relevance of data obtained. Sci. Rep. 6, 1-11 (2016).

65. Mosaad, E. O., Chambers, K. F., Futrega, K., Clements, J. A. \& Doran, M. R. The Microwell-mesh: A high-throughput 3D prostate cancer spheroid and drugtesting platform. Sci. Rep. 8, 1-12 (2018).

66. Zhang, L. et al. Tumorspheres derived from prostate cancer cells possess 
chemoresistant and cancer stem cell properties. J. Cancer Res. Clin. Oncol. 138, 675-686 (2012).

67. Al-Jamal, W. T. et al. Lipid-Quantum Dot Bilayer Vesicles Enhance Tumor Cell Uptake and Retention in Vitro and in Vivo. ACS Nano 2, 408-418 (2008).

\section{Acknowledgements}

This work was supported by the Prostate Cancer UK (Grant CDF12-002), the Engineering and Physical Sciences Research Council (EPSRC) (EP/M008657/1), and Slovenian Research Agency (Grant J3-7494, P3-0108). Authors would like to thank Professor A. Ganesan (University of East Anglia, Norwich) for his advice on Dox-PSA synthesis and Linda Strus for technical assistance with freeze-fracturing.

\section{Supporting Information}

MALDI-TOF spectra of Dox-PSA, RP-HPLC chromatogram of Dox-PSA, schemes of Dox-PSA loading techniques, Dox-PSA release, cytotoxicity of conventional liposomes. 
A

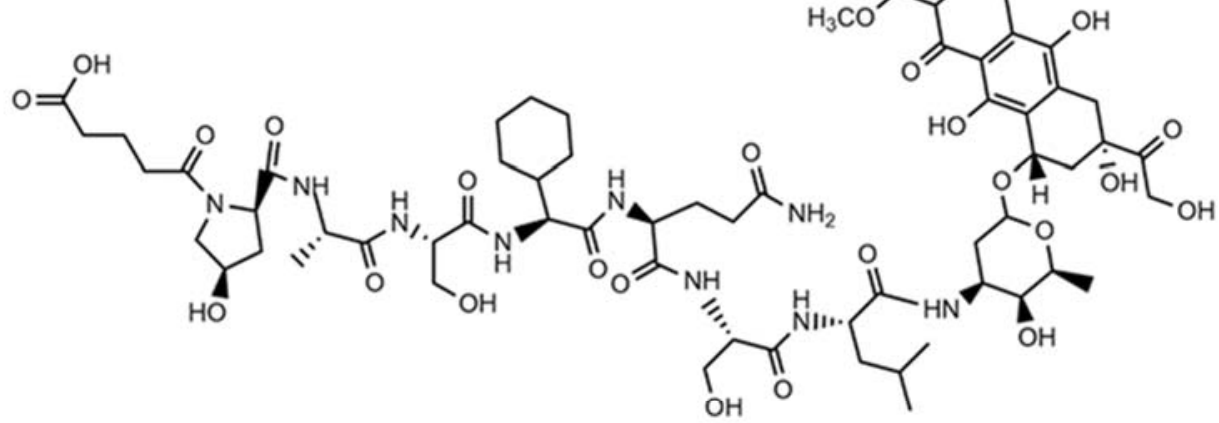

Glutaryl-Hyp-Ala-Ser-Chg-Gln-Ser-Leu-Dox

B

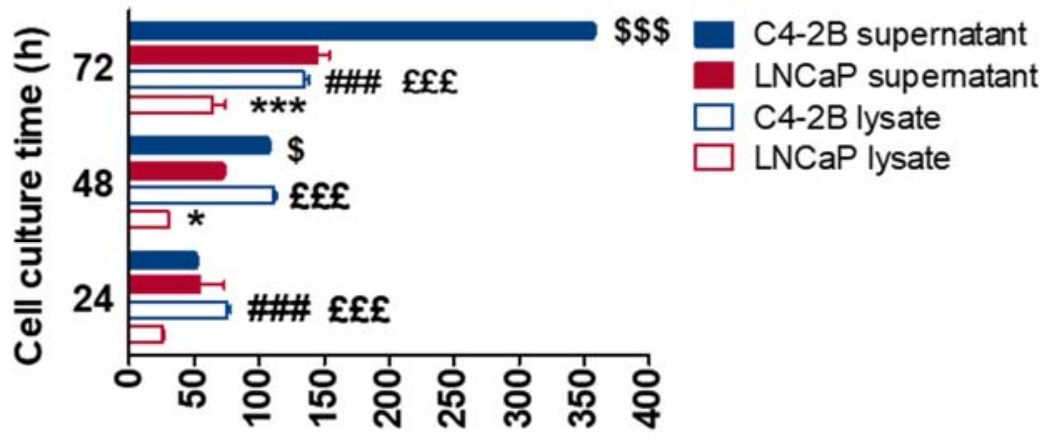

[Free PSA] (ng/mL $10^{5}$ cells)

C

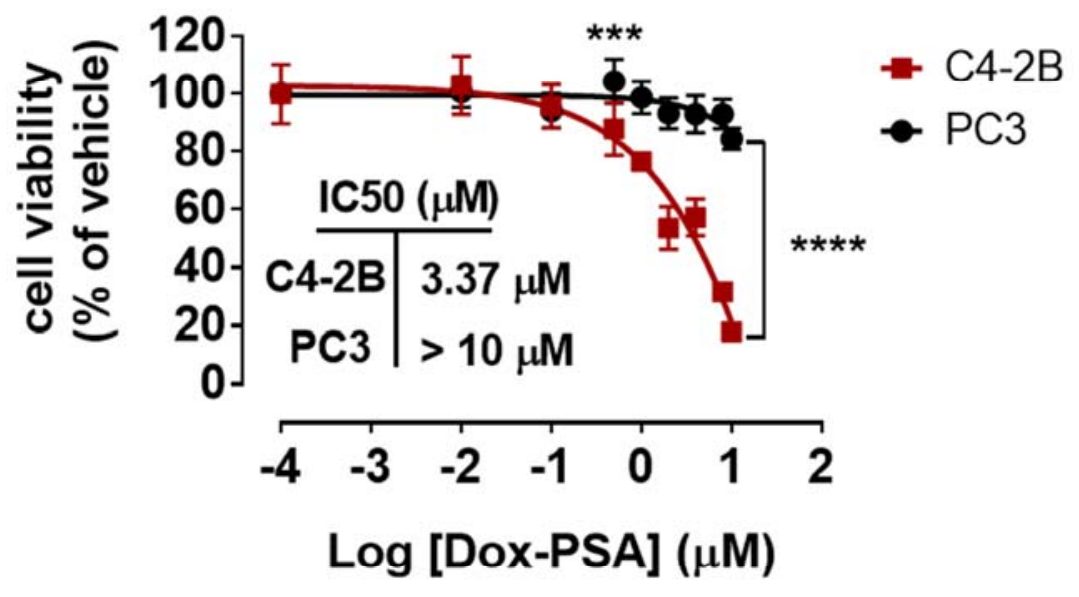

D

\begin{tabular}{|c|c|c|c|c|c|}
\hline \multicolumn{3}{|c|}{ C4-2B } & \multicolumn{3}{|c|}{ PC3 } \\
\hline Nuclei & Dox-PSA & Merge & Nuclei & Dox-PSA & Merge \\
\hline & & & 8 & 8 & 8 \\
\hline & ate & & & ? & 8 \\
\hline
\end{tabular}


Figure 1 | Dox-PSA is selective to PSA-expressing cells. (A) Dox-PSA chemical structure. (B) Free PSA levels in LNCaP and C4-2B cell lysates and supernatants. $10^{5}$ cells were seeded in 6-well culture plates and at 24, 48 and 72 hours post-incubation at $37^{\circ} \mathrm{C} / 5 \% \mathrm{CO}_{2}$, cell lysates and supernatants were collected for ELISA. Statistical significance was determined using two-way analysis of variance (ANOVA) followed by Bonferroni post-hoc test showing significant effects between LNCaP supernatant and C42B supernatant $\left({ }^{\$} \mathrm{p}<0.05,{ }^{\$ \$} \mathrm{p}<0.001\right)$, between $\mathrm{C} 4-2 \mathrm{~B}$ supernatant and C4-2B lysate $\left({ }^{\# \#} \mathrm{p}<0.001\right)$, between LNCaP lysate and C4-2B lysate $\left({ }^{\mathfrak{f f f}} \mathrm{p}<0.001\right)$, and between LNCaP supernatant and LNCaP lysate $\left({ }^{*} \mathrm{p}<0.05\right.$, ***p $\left.<0.001\right)$. (C) Viability of C4-2B (red squares) and PC3 (black circles) monolayers following treatment with free Dox-PSA. Cell viability was assessed by resazurin assay at 72 hours post-treatment. Insert show the corresponding $\mathrm{IC}_{50}$ values, obtained from the dose-response curves. Results are expressed as a percentage of vehicle-treated cells. Data shown as mean \pm SEM $(n=6)$. Statistical significance was determined using two-way analysis of variance (ANOVA) followed by Bonferroni post-hoc test showing significant effects between C4-2B and PC 3 cells treated with Dox-PSA $(* * * p<0.001, * * * * p<0.0001)$. (D) Cellular uptake of free Dox-PSA in PSA-secreting (C4-2B) and non-secreting (PC3) cells as visualised by CLSM. At 24 hours incubation, nuclear uptake is evident in C4-2B cells, which is not present in PC3 cells for at least 48 hours of incubation. Scale bar: $20 \mu \mathrm{m}$. 
A

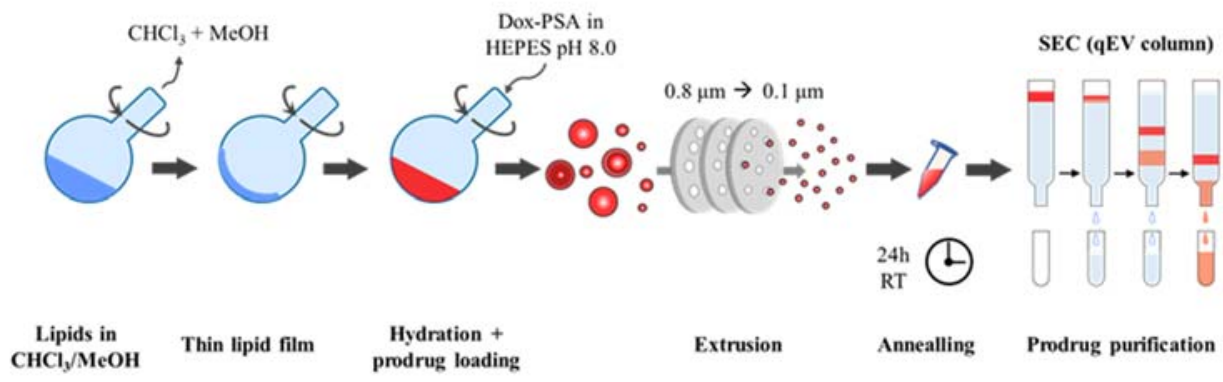

B

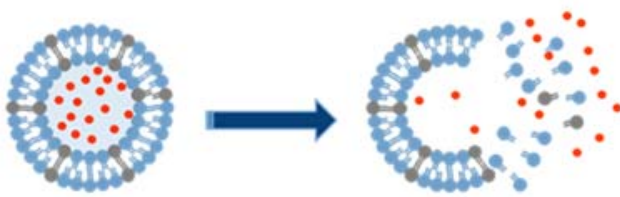

$\mathrm{pH} \geq 6.0 \quad \mathrm{pH} \leq 5.5$

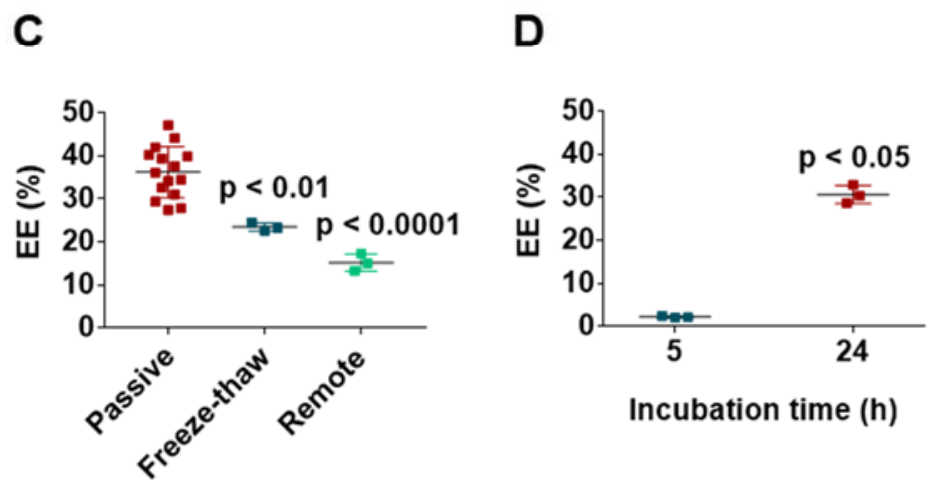

$\mathbf{E}$

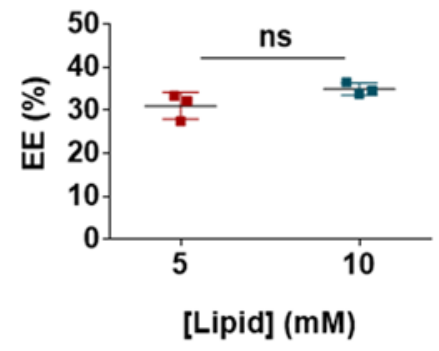

Figure 2 | Dox-PSA can be efficiently encapsulated inside DOPE:CHEMS liposomes.

(A) Scheme of the procedure used to prepare DOPE:CHEMS-Dox-PSA liposomes by passive loading of Dox-PSA. (B) Release mechanism of DOPE:CHEMS-Dox-PSA liposomes. Optimisation of Dox-PSA loading into DOPE:CHEMS liposomes. (C) DoxPSA loading methods. (D) Effect of Dox-PSA loading time on the encapsulation efficiency. (E) Effect of total lipid concentration on the encapsulation efficiency. Data are mean $\pm \mathrm{SD}(3 \geq \mathrm{n} \leq 15)$. 


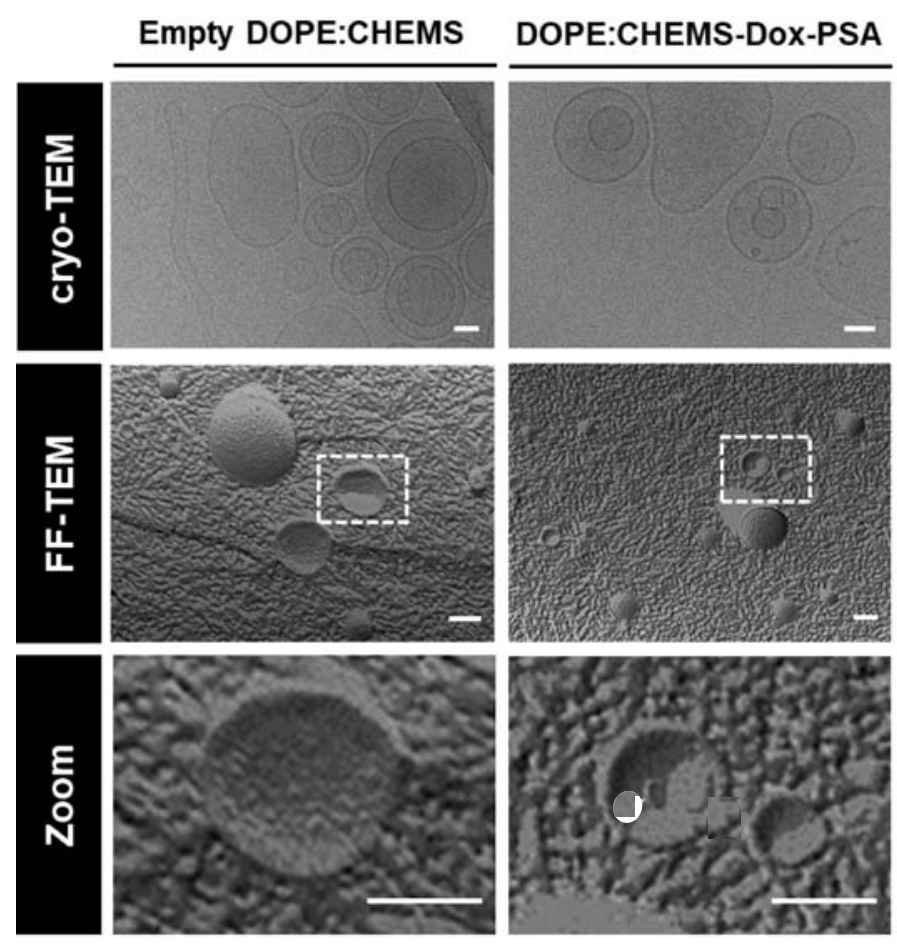

Figure 3 | Structural elucidation of Dox-PSA-loaded DOPE:CHEMS liposomes. Images of empty and Dox-PSA-loaded DOPE:CHEMS as shown by cryo-TEM (top panel) and freeze-fracture EM (FF-EM) (middle and bottom panels). Scale bar cryoTEM: $50 \mathrm{~nm}$, scale bar FF-EM: $100 \mathrm{~nm}$. 

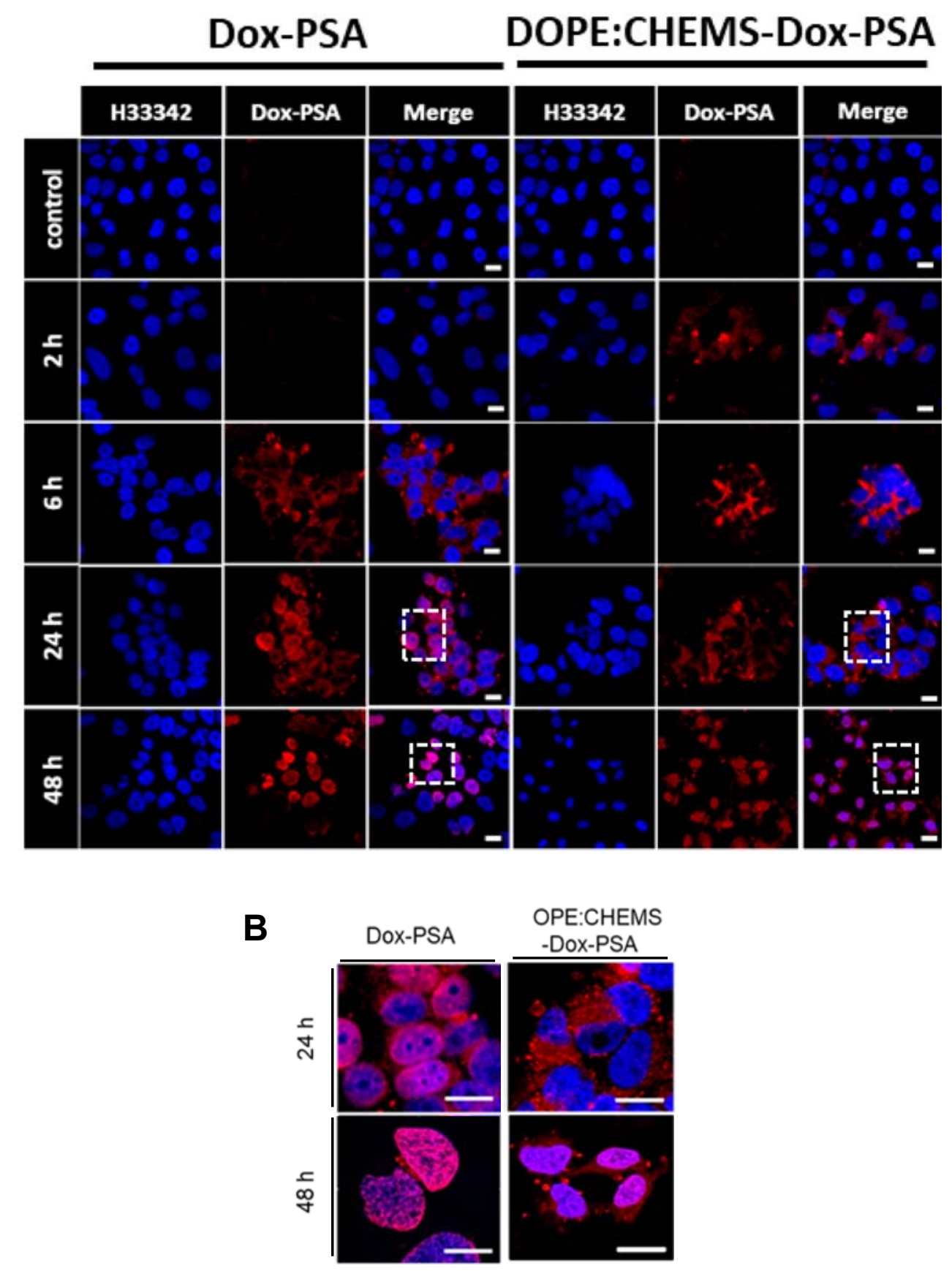

Figure 4 | DOPE:CHEMS-Dox-PSA liposomes are taken up by C4-2B cells, where the prodrug is released and activated. (A) Representative images showing the intracellular localisation of Dox-PSA and DOPE:CHEMS-Dox-PSA liposomes in C4-2B cells. Dox-PSA fluorescence (red) and the Hoechst 33342-stained nuclei of the cells (blue) were visualised by CLSM over 48 hours. Scale bar: $20 \mu \mathrm{m}$. (B) Magnified images showing the nuclear uptake of cleaved Dox 24 hours and 48 hours post incubation with free Dox-PSA and DOPE:CHEMS-Dox-PSA liposomes, respectively. Scale bar: $20 \mu \mathrm{m}$. 
A

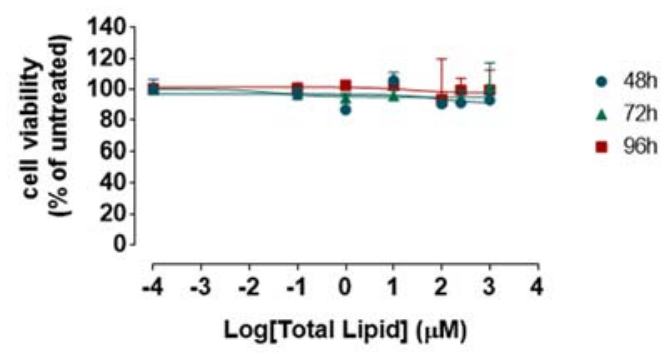

Dox-PSA

DOPE:CHEMS-DoX-PSA

B

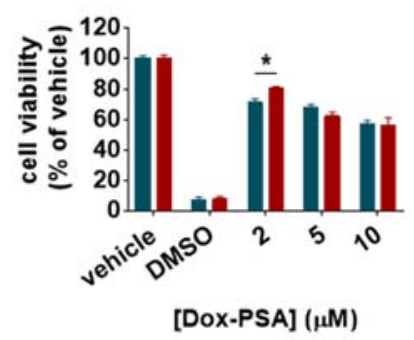

C

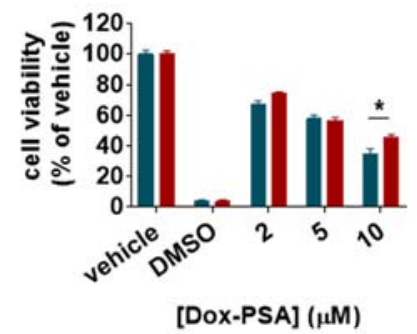

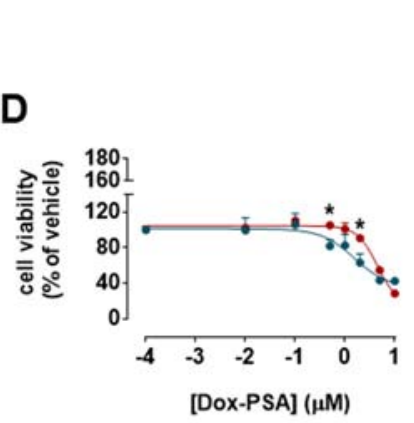

$\rightarrow$ Dox-PSA

$\rightarrow$ DOPE:CHEMS-DOX-PSA

E

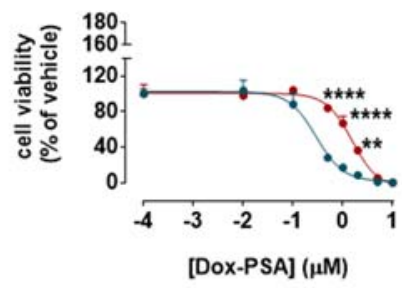

$\mathbf{F}$

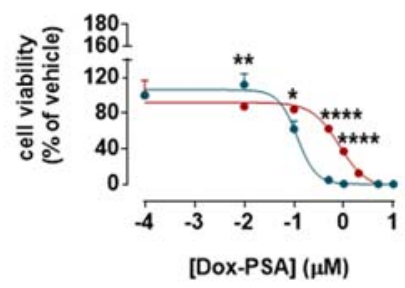

Figure 5 | DOPE:CHEMS-Dox-PSA liposomes maintain cytotoxic activity in C4-2B

monolayers. (A) Viability of $\mathrm{C} 4-2 \mathrm{~B}$ cells incubated with empty (prodrug-free) DOPE:CHEMS liposomes was assessed by resazurin assay up to 96 hours. Data are expressed as mean $\pm \mathrm{SD}(\mathrm{n}=3)$ of viable cells normalised to untreated cells. Statistical significance was determined using two-way analysis of variance (ANOVA) over untreated cells, with no statistically significant results found. C4-2B cells were treated with Dox-PSA (blue bars) or DOPE:CHEMS-Dox-PSA liposomes (red bars) for (B) 48 hours and (C) 72 hours. 10\% (v/v) DMSO was used as a positive control for toxicity. Cell viability was quantified by resazurin assay and normalised to the vehicle-treated cells. Data are expressed as mean $\pm \operatorname{SEM}(n=8)$. Statistical significance was determined 
using two-way analysis of variance (ANOVA) followed by Bonferroni post-hoc test showing significant effects between cells treated with free Dox-PSA and cells treated with DOPE:CHEMS-Dox-PSA liposomes $(* p<0.05)$. Dose-response viability curves of C4-2B monolayers treated with Dox-PSA (blue circles) or DOPE:CHEMS-Dox-PSA liposomes (red circles) for (D) 24 hours, (E) 48 hours, and (F) 72 hours. Impedance was continuously monitored using a Real-Time Cell Analyser (RTCA). Cell viability was normalised to the vehicle-treated cells. Data are expressed as mean $\pm \mathrm{SD}(\mathrm{n}=2)$. Statistical significance was determined using two-way analysis of variance (ANOVA) followed by Bonferroni post-hoc test showing significant effects between cells treated with Dox-PSA and cells treated with DOPE:CHEMS-Dox-PSA liposomes $(* p<0.05$, $* * \mathrm{p}<0.01, * * * * \mathrm{p}<0.0001)$. 
A

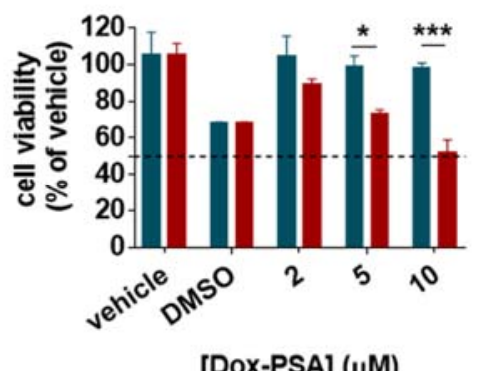

[Dox-PSA] $(\mu \mathrm{M})$
B

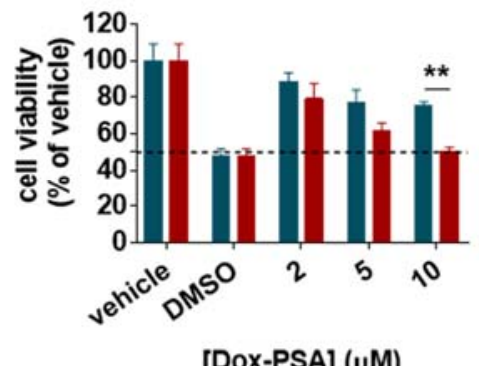

[Dox-PSA] ( $\mu$ M)
C

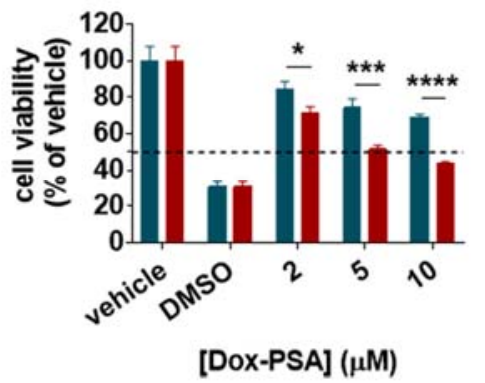

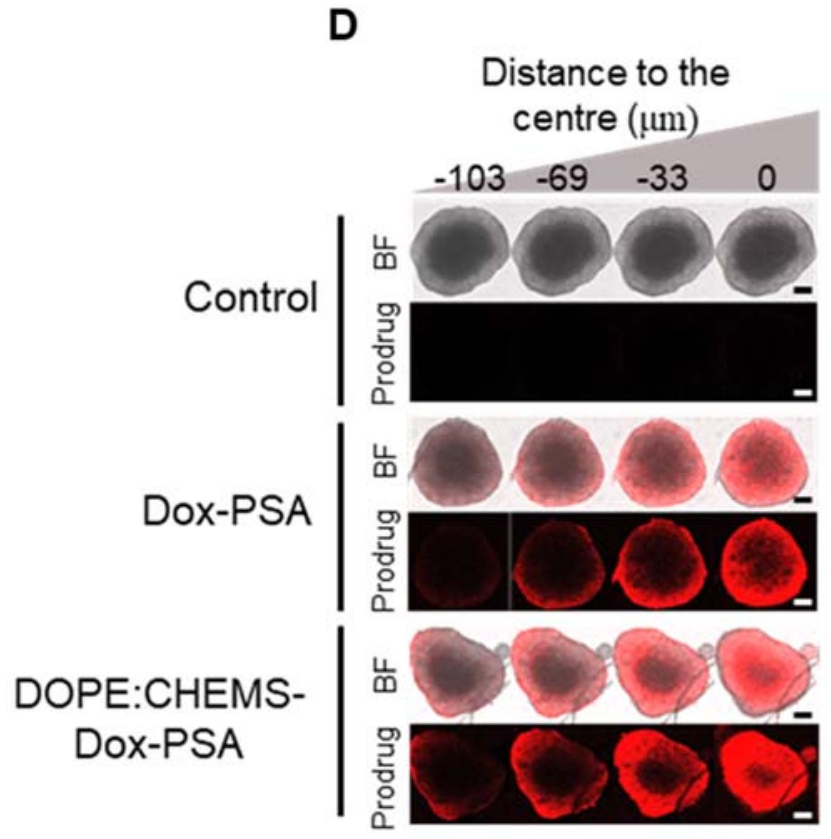

Figure 6 | DOPE:CHEMS-Dox-PSA show enhanced penetration and superior toxicity in C4-2B spheroids. C4-2B spheroids were treated with 2-10 $\mu \mathrm{M}$ Dox-PSA (blue bars) or DOPE:CHEMS-Dox-PSA liposomes (red bars) for (A) 48 hours, (B) 72 hours, and (C) 96 hours. 10\% (v/v) DMSO was used as a positive control for toxicity. Cell viability was quantified by a modified resazurin assay (details in the Methods section) and normalised to the vehicle-treated spheroids. Data are expressed as mean \pm SEM $(n=4)$. Statistical significance was determined using two-way analysis of variance (ANOVA) followed by Bonferroni post-hoc test showing significant effects between cells treated with free Dox-PSA and cells treated with DOPE:CHEMS-Dox-PSA liposomes $\left({ }^{*} \mathrm{p}<0.05, * * \mathrm{p}<0.01,{ }^{* * *} \mathrm{p}<0.001, * * * * \mathrm{p}<0.0001\right)$. (D) Bright field and fluorescent images depicting the penetration of Dox-PSA and DOPE:CHEMS-Dox-PSA over 48 hours in C4-2B spheroids. Scale bar: $100 \mu \mathrm{m}$. 
Table of content

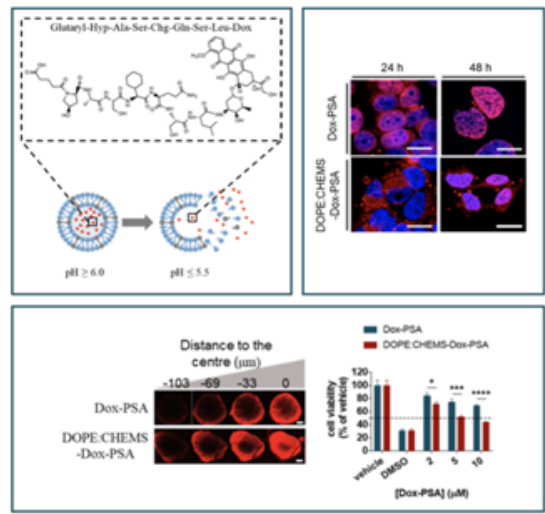

